\title{
Apparent diffusion coefficient restriction in the white matter: going beyond acute brain territorial ischemia
}

\author{
Valentina Citton • Alberto Burlina • Claudio Baracchini • Massimo Gallucci • \\ Alessia Catalucci $\cdot$ Sandro Dal Pos • Alessandro Burlina $\cdot$ Renzo Manara
}

Received: 11 November 2010 / Revised: 9 May 2011 /Accepted: 28 June 2011 / Published online: 20 December 2011

(C) European Society of Radiology 2011

\begin{abstract}
Background Reduction of apparent diffusion coefficient (ADC) values in white matter is not always ischaemic in nature. Methods We retrospectively analysed our MRI records featuring reduced $\mathrm{ADC}$ values in the centrum semiovale without grey matter involvement or significant vasogenic oedema. Results Several conditions showed the aforementioned MR findings: moose-horn lesions on coronal images in X-linked Charcot-Marie-Tooth disease; small fronto-parietal lesions in Menkes disease; marked signal abnormalities in the myelinised regions in the acute neonatal form of maple syrup urine disease; strip-like involvement of the corpus callosum in glutaric aciduria type 1; persistent periventricular parietooccipital abnormalities in phenylketonuria; diffuse signal abnormalities with necrotic evolution in global cerebral
\end{abstract}

V. Citton $(\bowtie) \cdot$ S. Dal Pos $\cdot$ R. Manara

University of Padua, Neuroradiological Unit,

Padova, Italy

e-mail: valentina.citton@sanita.padova.it

C. Baracchini

Department of Neurological Sciences,

University Hospital of Padua,

Padua, Italy

M. Gallucci $\cdot$ A. Catalucci

Neuroradiologic Unit, University of L'Aquila,

L'Aquila, Italy

A. Burlina

Division of Neurology,

San Bassiano Hospital of Bassano del Grappa,

Padua, Italy

\section{A. Burlina}

Department of Pediatrics, University of Padua, Padua, Italy anoxia or after heroin vapour inhalation; almost completely reversible symmetric fronto-parietal lesions in methotrexate neurotoxicity; chain-like lesions in watershed ischaemia; splenium involvement that normalises in reversible splenial lesions or leads to gliosis in diffuse axonal injury.

Conclusion Neuroradiologists must be familiar with these features, thereby preventing misdiagnosis and inappropriate management.

Keywords Apparent diffusion coefficient (ADC) values . MRI $\cdot$ DWI

\section{Introduction}

Diffusion-weighted imaging (DWI) is a relatively new technique in which the image contrast mostly depends on the random microscopic motion of water molecules in tissues. DWI has been primarily applied in acute brain ischaemia, which is characterised in its early phase by cytotoxic oedema. Thanks to its sensitivity in detecting hyperacute ischaemic strokes, DWI has gained a primary role in routine brain MR protocols when dealing with acute neurological syndromes. The frequent utilisation of DWI has unexpectedly revealed peculiar signal abnormalities in several other cerebral diseases, such as abscesses, tumour and head trauma, widening even further the spectrum of brain diseases where this sequence helps to address the diagnosis. The reduction of the apparent diffusion coefficient (ADC) values depends on the disease studied, and it might involve an increase in cell debris, cellularity, intramyelin and axonal oedema.

The neuroradiologist is often challenged by neurologists and paediatricians with neuroimaging studies that document white matter lesions characterised by ADC restriction. 
In this pictorial we focused on different conditions (pertaining to adult or child neurology) other than territorial ischaemic stroke where DWI signal abnormalities and ADC value reduction are confined within the white matter.

The DW images throughout the study were performed with a b value of 800 or $1,000 \mathrm{~s} / \mathrm{mm}^{2}$.

Genetic and metabolic disorders

X-linked Charcot-Marie-Tooth disease (CMTX) is the second most common form of inherited motor and sensory demyelinating neuropathy next to the CMT Type 1A; it accounts for approximately $10-20 \%$ of all hereditary demyelinating neuropathies. It is associated with different mutations in the protein connexin 32 (CX32), a gap junction protein widely expressed in Schwann cells, oligodendrocytes and neurons [1,2]. Even if this disease is considered a disorder of the peripheral nervous system, some CX32-mutated subjects might present with acute onset of central nervous system dysfunctions such as hemiparesis, ataxia or aphasia associated with transient white matter changes in the brain. In particular, neuroradiological MR findings in the acute phase show symmetrical, largely confluent cerebral deep white matter signal abnormalities, predominantly in the posterior centrum semiovale, the posterior limb of the internal capsule, the splenium of the corpus callosum (Fig. 1) and sometimes in the middle cerebellar peduncles [1-3]. Characteristically, $U$ fibres are spared and the lesions do not demonstrate contrast enhancement [3]. Bilateral T2 and DWI signal abnormalities might disappear within 1 week or might persist for several months, unrelated to the clinical picture that often appears clearly asymmetric and usually improves rapidly.

As ADC reduction might reverse after a few months, signal abnormalities cannot be interpreted as simple cytotoxic oedema, which usually lasts less than 2 weeks, while the increase in the magnetisation transfer ratios is not consistent with demyelination or oedema. As gap junction dysfunctions may disrupt the diffusion of small molecules and ions across myelin sheaths, the more likely explanation of the reversible white matter lesions remains myelin splitting and intra-myelin oedema with compression of the extracellular spaces [4].

Maple syrup urine disease (MSUD) is a rare autosomic recessive disorder caused by a deficiency of the branchedchain $\alpha$-keto acid dehydrogenase mitochondrial multienzyme complex. A sudden metabolic decompensation with encephalopathy occurs spontaneously or in concomitance with minor illnesses; high concentrations of upstream metabolites are thought to exert the neurotoxic effect. Clinical presentation and outcome are heterogeneous; in the classical phenotype the prognosis is very poor, and untreated surviving children are left with psychomotor retardation, spasticity, generalised dystonia and cerebral blindness. In the classical neonatal form, which accounts for about $80 \%$ of cases [4], brain MRI may provide a highly suggestive pattern of lesions with a generalised cerebral oedema and a striking increase in DWI and T2 signal intensity in all areas that are already myelinated at birth (Fig. 2). At microscopic examination the same areas represent myelin splitting and intra-myelin vacuoles. These peculiar histological changes are thought to explain the observed reduction in ADC values and its reversibility. Early diagnosis and treatment may normalise clinical and neuroradiological abnormalities, although inter-current illnesses throughout life may lead to further metabolic derangements [4].

Menkes disease is an $\mathrm{X}$-linked disorder caused by defective copper-transporting adenosine triphosphatase, which results in low levels of intracellular copper. Male children are normal in the first 2-4 months of life, and then present with loss of developmental milestones, hypotonia, seizures and failure to thrive; these patients usually have short, hypopigmented, kinked hair; death often occurs early in childhood. Brain MRI might disclose diffuse brain atrophy, subdural effusion, or haematoma and vascular
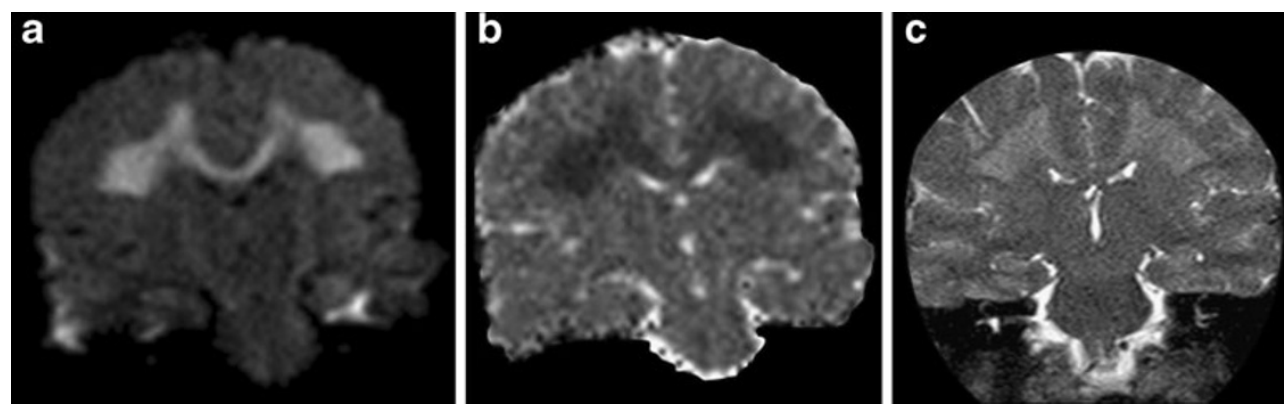

Fig. 1 A 23-year-old male patient affected by genetically proven Xlinked Charcot-Marie-Tooth disease presenting with acute onset of aphasia and right hemiparesis. Diffusion-weighted (a) and T2-weighted images (c) at admission showed symmetrical confluent hyperinten- sities in the posterior centrum semiovale and in the splenium of the corpus callosum, with a coronal image resembling "moose horns". Apparent diffusion coefficient (ADC) maps (b) disclosed a restricted diffusion of water molecules in the corresponding areas 
Fig. 2 Diffusion-weighted axial images (superior row) and ADC axial images (inferior row) obtained in a newborn with a biochemically confirmed classical form of maple syrup urine disease. He was admitted with severe encephalopathy and coma. The myelinated areas shown as symmetrical, markedly high intensity signal on a rather homogeneous brain parenchyma depict the corticospinal tract $(\mathbf{a}, \mathbf{b})$ and the cerebello-pontine white matter (c). Restricted diffusion of water molecules is well demonstrated by ADC images $(\mathbf{d}, \mathbf{e}, \mathbf{f})$
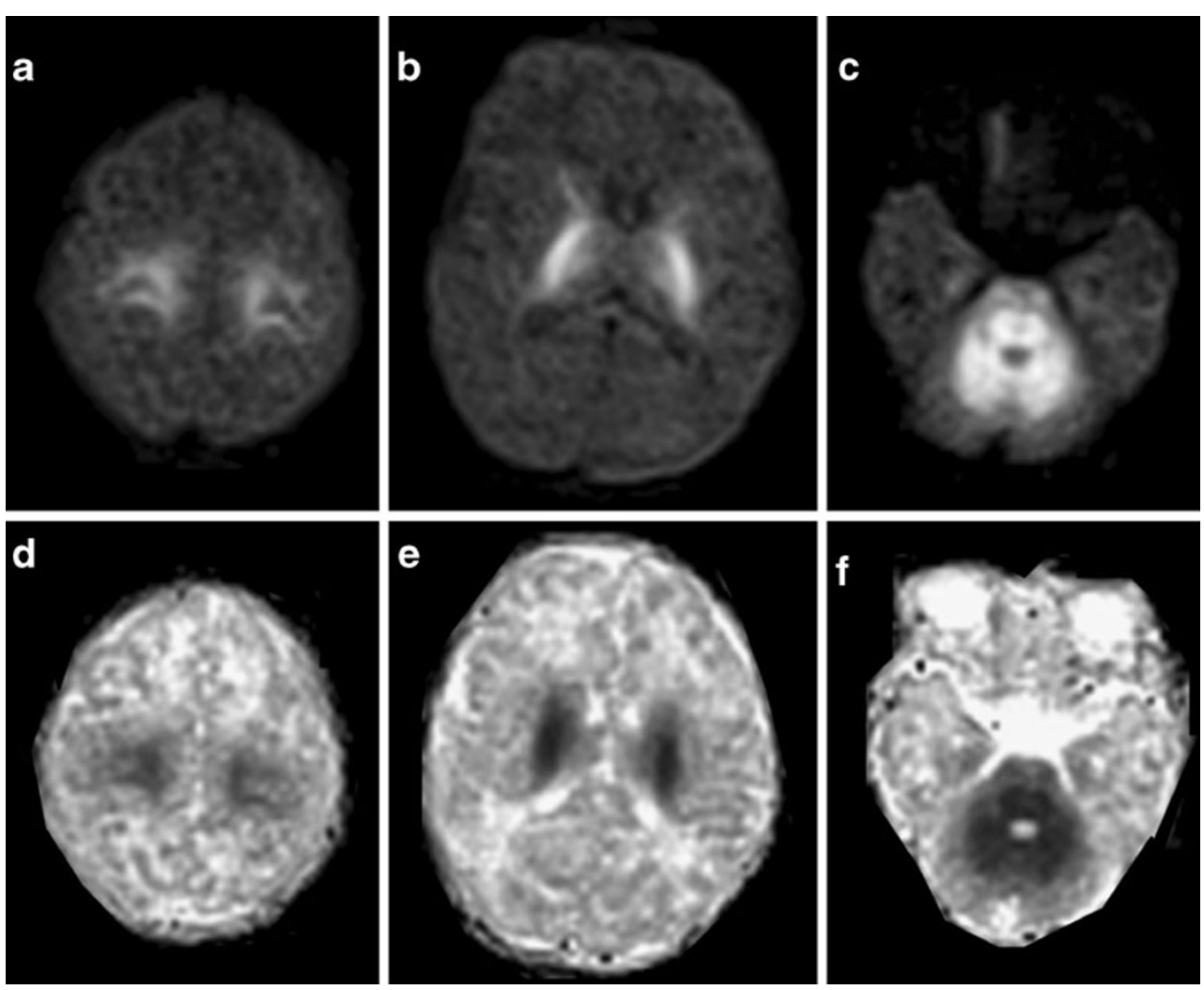

tortuosity. Asymmetrical localised white matter lesions involving lobar white matter have been described $[5,6]$. Recently, Lee et al. [7] reported that white matter lesions are bright on DWI and present decreased ADC values consistent with cytotoxic oedema (Fig. 3).

As in CMT and MSUD disease, the pathogenesis of these findings is uncertain. According to Hsich et al. [8], the tortuosity of the vessels might produce erratic and turbulent blood flow, thus predisposing to deep white matter ischaemia [8]. Alternatively, brain tissue abnormalities may be related to a deficiency in ATP7A mitochondrial copper-containing enzyme [9] with consequent energy failure.

Glutaric aciduria type 1 (GA-1) is a rare autosomal recessive disorder caused by deficiency of the mitochondrial enzyme glutaryl CoA dehydrogenase, necessary for the metabolism of three essential amino acids: lysine, hydroxylysine and tryptophan. The metabolic blocking of these amino acids results in increased levels of glutaric acid and 3-hydroxy glutaric acid. Because excessive glutaric acid is probably toxic to the striatal cells, atrophy in these nuclei occurs with age [10]. Patients may be asymptomatic or present with acute metabolic decompensation and striatal necrosis, which often leads to severe dyskinesia and cognitive impairment.

Magnetic resonance imaging findings in GA-1 include wide cerebrospinal fluid spaces anterior to the temporal lobes and within the sylvian fissure [11], subdural collections and brain atrophy. Oedema and increased T2 signal intensity within both caudate heads and lentiform nuclei are
Fig. 3 An 8-month-old child affected by Menkes disease. Axial diffusion- (a) and T2weighted images (c) disclosed bilateral asymmetric oval bright white matter lesions in the centrum semiovalis (arrows). ADC maps (b) of the lesions showed low intensity signal
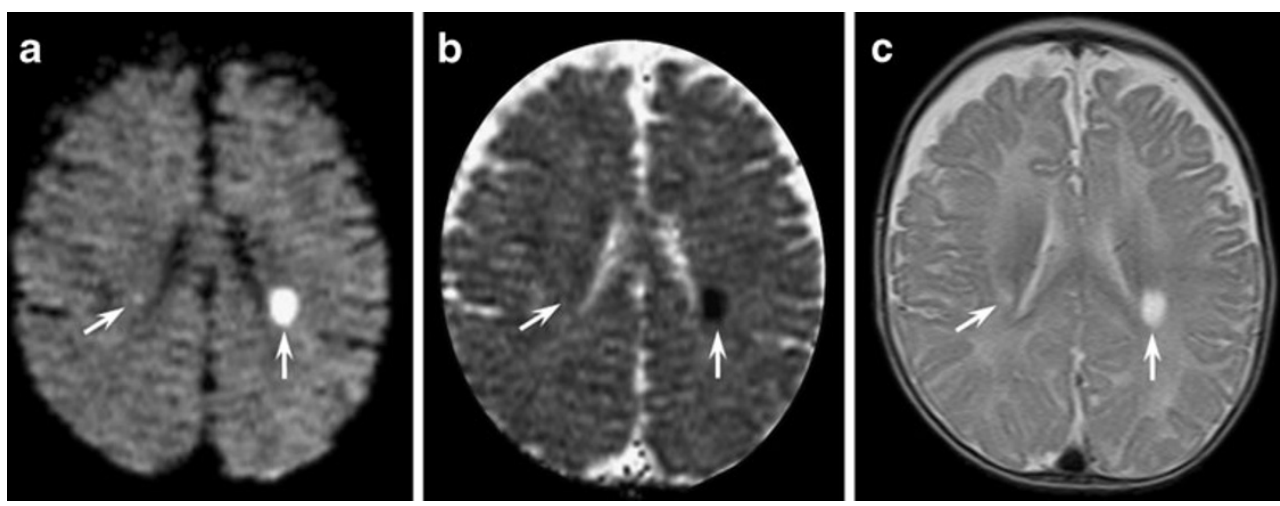
Fig. 4 Axial diffusion- (a) and T2-weighted images (c) of an asymptomatic 26-year-old patient with type 1 glutaric aciduria demonstrating the peculiar strip-like involvement of the corpus callosum due to signal abnormalities in the median genu of the corpus callosum (arrows) and in periventricular white matter (arrowhead). ADC maps (b) revealed mildly reduced values in the affected areas
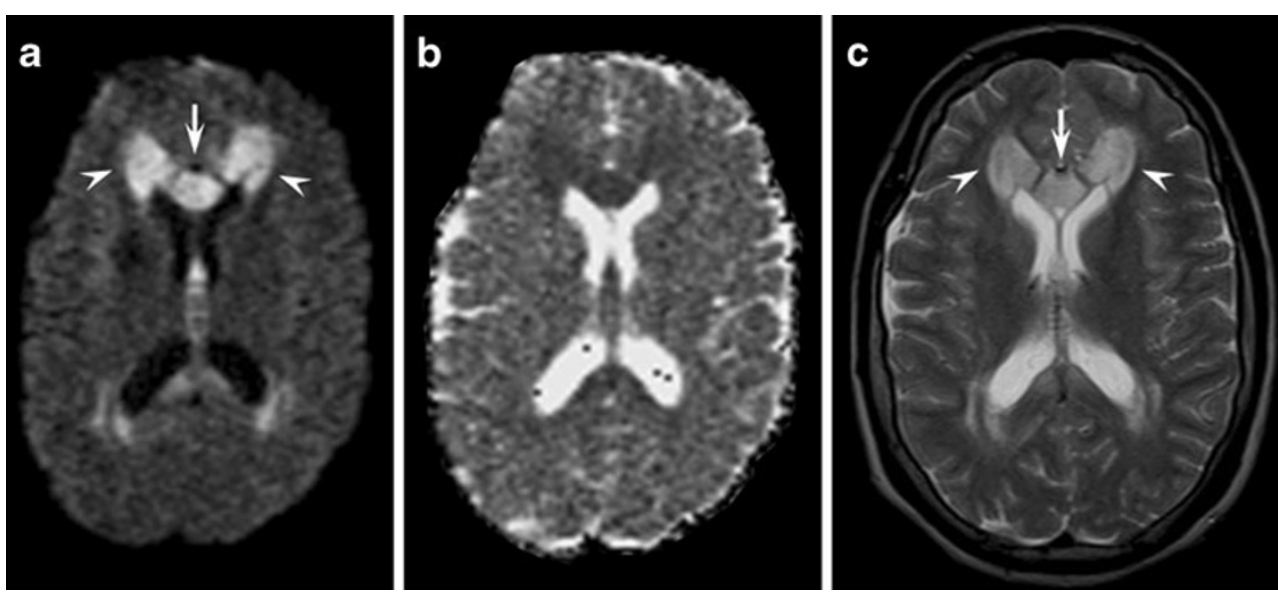

seen during metabolic derangement followed by necrosis and shrinking of these structures [10].

In older children as well as in asymptomatic patients, a peculiar and persistent DWI hyperintensity of white matter with a peculiar strip-like involvement of the corpus callosum has been noted. Within white matter lesions, ADC values are reduced (Fig. 4).

No convincing, definitive explanation has yet been found for the long persistence of these signal abnormalities. Spongiform changes in the white matter sparing $U$ fibres have been demonstrated histopathologically [10].

Phenylketonuria (PKU) is a severe autosomal recessive disorder due to phenylalanine hydroxylase deficiency [4, $12,13]$ that strongly benefits from early diagnosis and dietary treatment. Brain MR examination of adolescents and adults discloses white matter abnormalities both in untreated PKU patients and those treated early.

Typically, white matter lesions are occipital-parietal periventricular signal abnormalities on T2 sequences and are related to phenylalanine blood concentrations without significant correlation with the neurological impairment.

Recently, restricted diffusion of water molecules has been reported in the areas of white matter abnormalities $[4$,
$12,14,15]$, appearing hyperintense on DWI in an otherwise rather homogeneously grey brain parenchyma (Fig. 5). These findings have been interpreted as cytotoxic oedema because of a reduction in the $\mathrm{Na}+\mathrm{K}+$-ATPase activity [16] or alternatively as dysmyelination with intra-myelin oedema [16], but the long persistence of the signal abnormalities does not support these hypotheses [16].

\section{Vascular conditions}

Watershed infarctions account for $0.7-3.2 \%$ of all strokes [17] and may be confined to the deep white matter. In the acute phase, MR examination may reveal peri-ventricular round foci of restricted diffusion, with a "string of pearls appearance" within the deep white matter (Fig. 6) [17]. Despite a multifocal bilateral appearance, all these lesions functionally represent a single vascular territory between superficial branches and deep perforators of cerebral arteries. This territory is particularly vulnerable in conditions of severely impaired cerebral blood flow such as prolonged hypotensive events, cardiac arrest with resuscitation and Moyamoya disease.

On MRI lesions might also be unilateral in the case of an ipsilateral internal carotid artery occlusion or tight stenosis
Fig. 5 Magnetic resonance images obtained in an asymptomatic, phenylketonuric adult who was treated early (plasmatic phenylalanine levels of $729 \mu \mathrm{mol} / \mathrm{l}$ n.v. $44-66 \mu \mathrm{mol} / 1)$. Axial diffusion-weighted (a) ADC maps (b) and T2-weighted images (c) showed periventricular white matter signal abnormalities predominantly in the parieto-occipital regions (arrows)
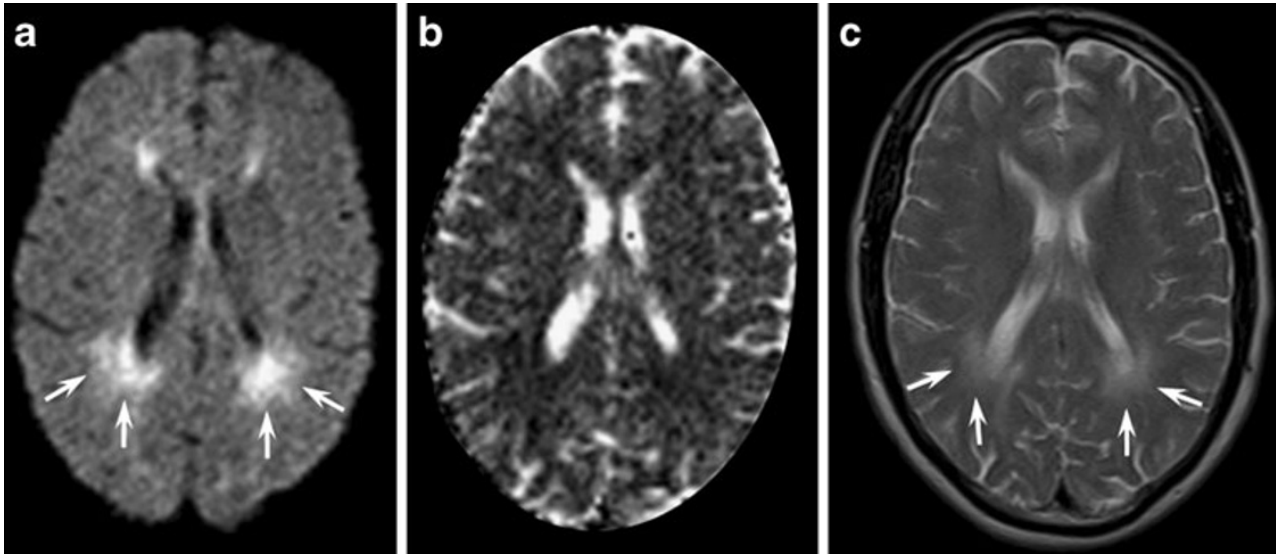
Fig. 6 A 53-year-old male patient affected by dissection of the left internal carotid artery. Axial diffusion (a) and T2weighted images (c) revealed hyperintense foci in the subcortical watershed territories in a characteristic parasagittal chainlike distribution (arrows). ADC maps disclosed the cytotoxic nature of the lesions (b)
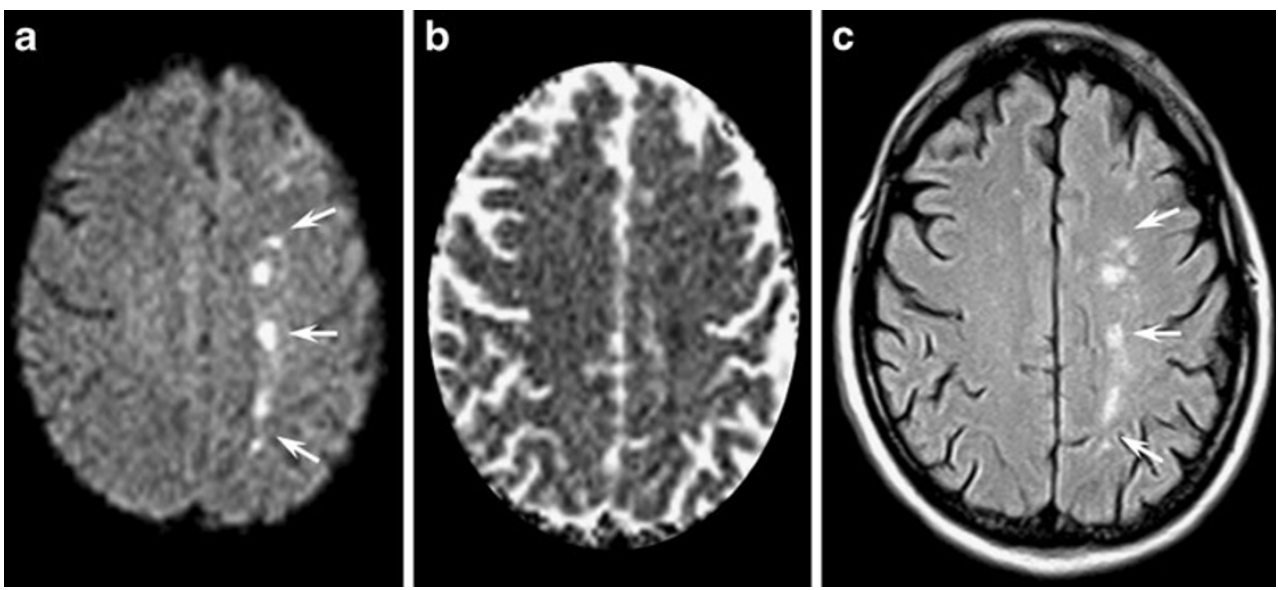

or main intracranial artery stenosis, especially when intracranial collateral flow is not compensatory (e.g. because of the incompleteness of the circle of Willis).

Contrary to patients with territorial infarction, patients with WI often do have a history of transient ischaemic attacks, generally have better outcomes, and usually do not benefit from thrombolysis.

Grey matter is known to be more vulnerable to diffuse hypoxic-ischaemic injury than neighbouring white matter [18], but recent reports have highlighted that white matter is more sensitive to ischaemia than previously thought and may be primarily involved, sometimes in an isolated fashion [19, 20]. Global hypoxic-ischaemic encephalopathy of the adult is generally secondary to prolonged cardiac arrest, cardiac arrhythmias, drug overdose, respiratory failure or carbon monoxide poisoning [21].

Even though prominent symmetrical, restricted diffusion abnormalities (Fig. 7) have been described in the early stages of diffuse hypoxic-ischaemic injury [21], white matter abnormalities typically appear in the late-subacute period (14-20 days) and may normalise thereafter.

Histologically, diffuse white matter abnormalities on MR imaging have correlated with deep white matter vacuolar degeneration and with adjacent axonal injury in normalappearing white matter [22].

The outcome is generally poor with only a minority of patients regaining independent function [23].

Iatrogenic conditions

Pontine and extrapontine myelinolysis is a rare condition elicited by hypo- or hyper-natraemia, especially when an inappropriate (i.e. too fast) correction occurs.

Magnetic resonance imaging might show a characteristic lesion, namely a trident-shaped signal change within the pons with sparing of the tegmentum (pontine myelinolysis) or symmetrical bilateral signal changes of the middle cerebellar peduncles, of the cortico-spinal tract and of the
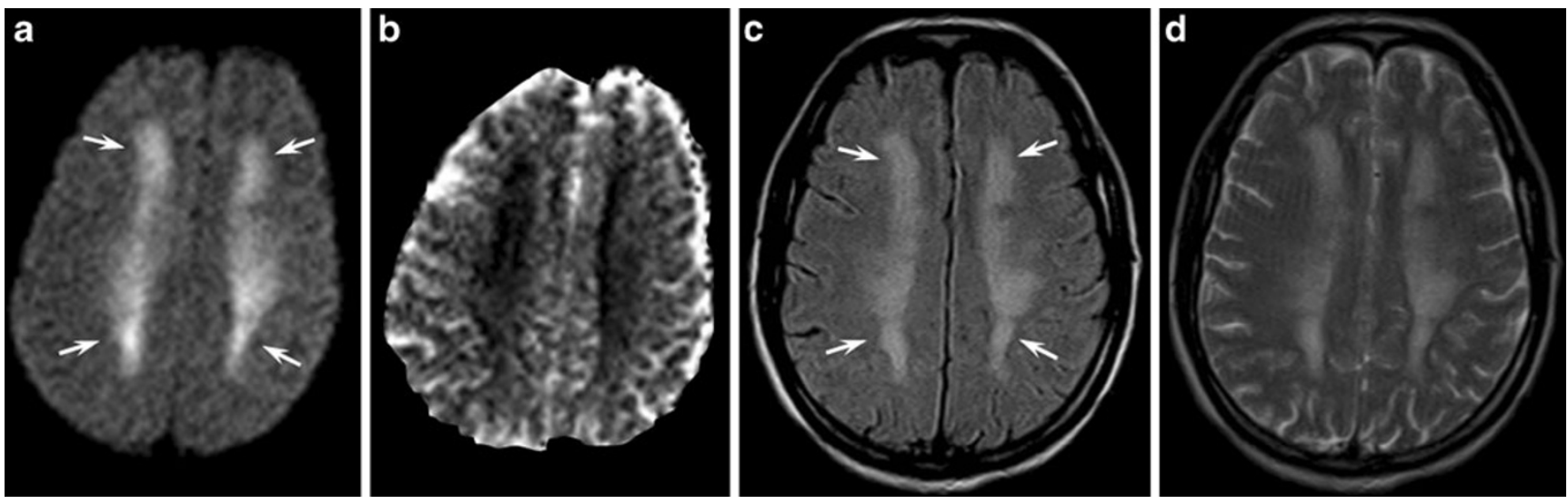

Fig. 7 Magnetic resonance images obtained during the subacute stage of $\mathrm{CO}$ intoxication in a 47-year-old man with a history of heroin abuse found unconscious at home. Diffusion-weighted (a), FLAIR (c) and T2-weighted images (d) disclosed prominent, bilateral and symmetrical confluent hyperintensities in the centrum semiovalis that were

more evident in the fronto-parietal lobes (arrows). On ADC maps (b), white matter lesions appeared moderately hypointense due to reduced water diffusion. As CO intoxication and heroin-associated spongiform leukoencephalopathy may produce similar MRI findings, both events could have resulted in this peculiar MR picture in our patient 
splenium of the corpus callosum (extrapontine myelinolysis) (Fig. 8) [17]. It might be difficult to differentiate extrapontine myelinolysis from Wallerian degeneration [24]. In fact, several case reports of pontine stroke present with signal abnormalities in the middle cerebellar peduncles [25]. However, in Wallerian degeneration signal abnormalities are usually fainter compared with those of extrapontine myelinolysis. Furthermore, an associated pontine lesion is the key to a definitive diagnosis of Wallerian degeneration of the ponto-cerebellar tracts [26].

Despite the term "myelinolysis", several cases of bilateral basal ganglia involvement have been reported.
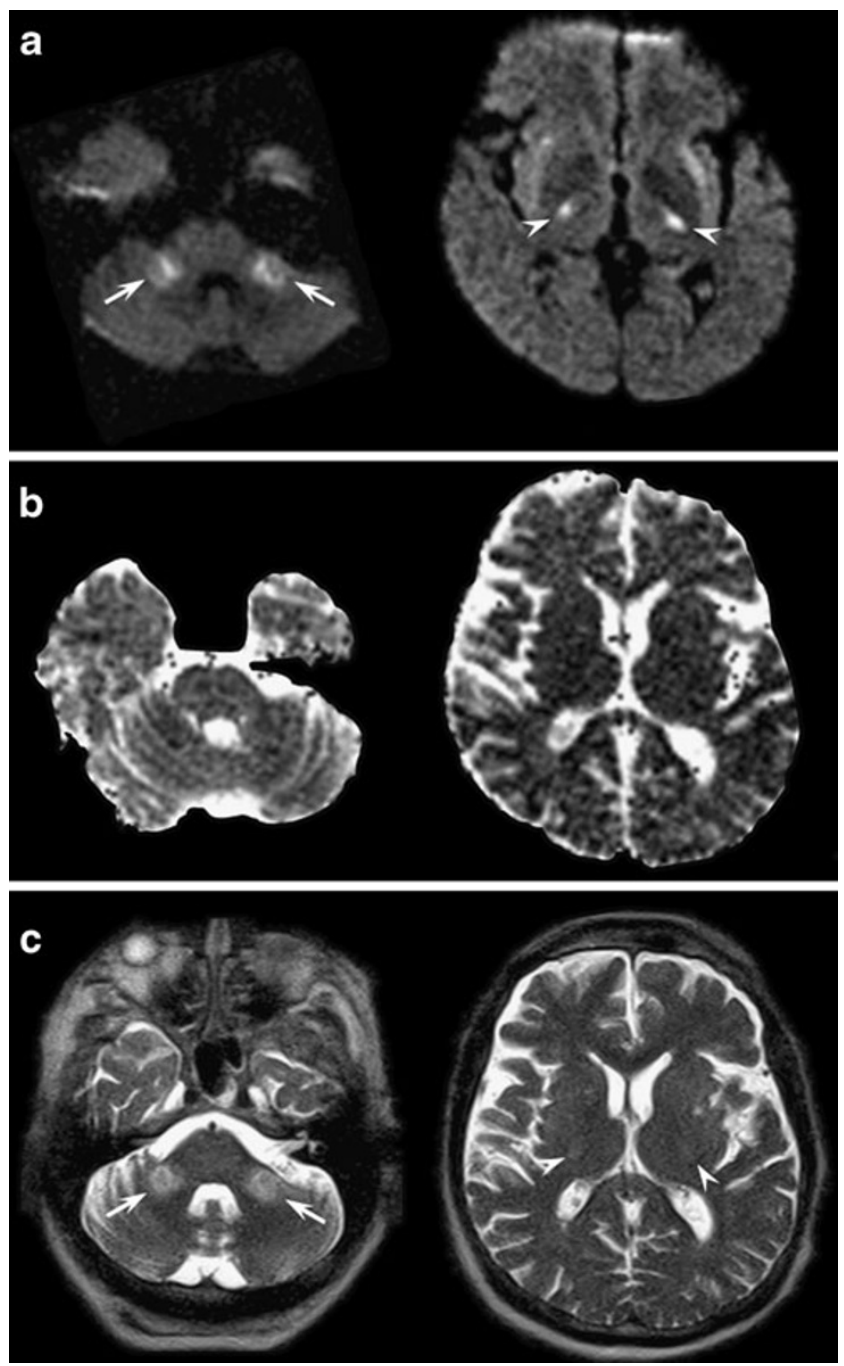

Fig. 8 Axial diffusion-weighted (a), ADC maps (b) and T2-weighted (c) images at the level of the pons (left images) and basal ganglia (right images) obtained in a 54-year-old man who developed impaired consciousness after rapid correction of severe hyponatraemia. Diffusionweighted and T2-weighted images revealed white matter hyperintensities that were more evident in the middle cerebellar peduncles (arrows) and in the cortico-spinal tract (arrowheads) consistent with osmotic extrapontine myelinolysis. ADC values were mildly decreased
DWI may show a variable decrease in ADC values, which probably depends on the timing of the MR examination and on the severity of white matter involvement.

Following an initial stage of diffuse encephalopathy, the neurological sequelae are usually characterised by diffuse cognitive deficits and extrapyramidal or cortico-bulbospinal signs; however, a complete remission of neurological symptoms can also be achieved. Variable T2 hyperintense lesions with increased ADC values are usually found on follow-up examinations, although complete regression of the MR lesions is possible.

Methotrexate (MTX) is a cycle-specific antimetabolite that inhibits the enzyme dihydrofolate reductase and is used for the treatment of oncological disorders. MTX crosses the bloodbrain barrier and can be administered intravenously as well as intrathecally to eradicate leukaemic cells from the CNS and to prevent CNS recurrence in acute lymphocytic leukaemia. Acute MTX neurotoxicity has been described in 3-10\% of cases and varies with dose, frequency and route of administration [27-29]. The temporal relationship between acute MTX neurotoxicity and induction ranges from 2 to 127 weeks [28], but it is most often seen 10-11 days after intrathecal administrations of MTX. From a clinical point of view acute MTX neurotoxicity may mimic a stroke and present with aphasia or hemiparesis. Conventional MR imaging shows bilateral $\mathrm{T} 2$ hyperintense lesions within the deep fronto-parietal white matter [28]. DWI identifies the white matter involvement earlier than other sequences (Fig. 9) and reveals a variable decrease of ADC values probably due to the derangement of folate and homocysteine metabolism. DWI abnormalities and clinical symptoms usually resolve within a few days even when T2 abnormalities persist.

\section{Non-iatrogenic toxicity}

Inhalation of heroin vapour, a practice known as "chasing the dragon", can cause a specific leukoencephalopathy. The pathophysiological mechanism seems to be selectively activated by substances originating during the heating of heroin. Symptoms usually occur within some days after the last heroin consumption and are first dominated by cerebellar deficits.

Characteristically, heroin-induced leukoencephalopathy appears as symmetrical T2 and DWI hyperintense lesions involving the cerebellar white matter, the posterior limbs of the internal capsule, the splenium of the corpus callosum and the posterior cerebral white matter [30]. Sub-cortical U fibres are usually spared (Fig. 7) [30]. A decrease in the ADC values and the absence of contrast enhancement further characterise the affected areas, whereas a spongiform demyelination is considered the histological substrate. Clinical and neuroradiological pictures may slowly improve if the patients are treated promptly; otherwise manifest severe neurological impairment and death occur. 

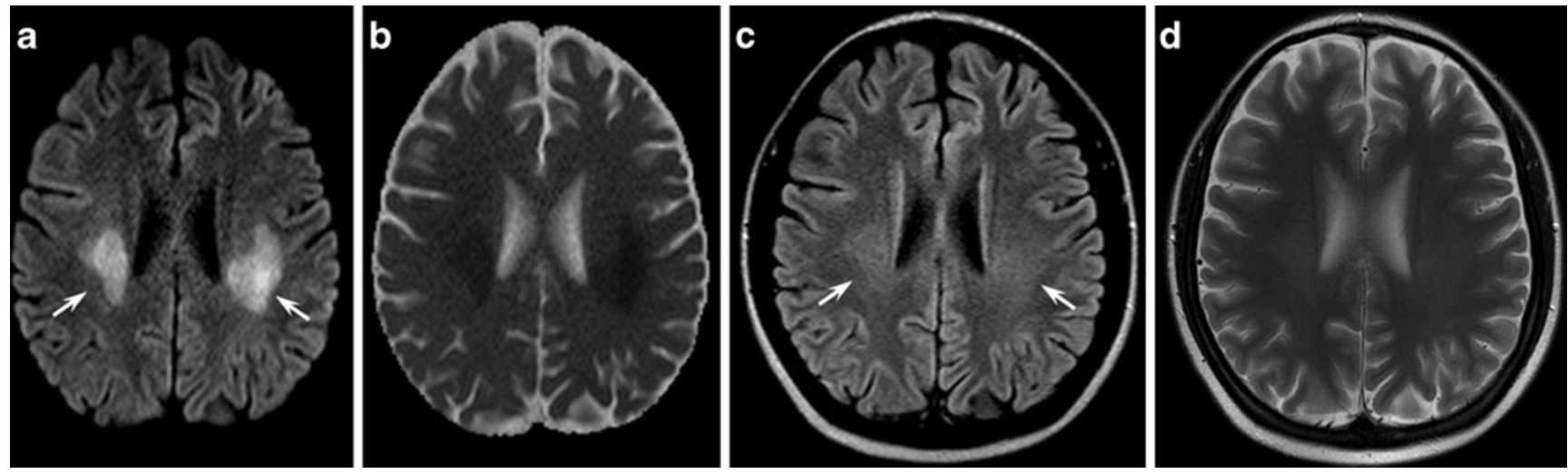

Fig. 9 This 14-year-old girl affected by acute lymphocytic leukaemia and treated with intrathecal MTX acutely developed hemiparesis and aphasia. Axial diffusion-weighted (a), FLAIR (c) and T2-weighted images (d) showed bilateral T2 hyperintense lesions within the deep

\section{Traumatic condition}

Diffuse axonal injury (DAI) accounts for $50 \%$ of all primary intra-axial traumatic brain injuries. These lesions can be both haemorrhagic and non-haemorrhagic, and are caused by differential linear and rotational accelerations of the cortex in relation to the underlying deep brain structures.

Besides multi-focal punctuate haemorrhages at the fronto-temporal cortico-medullary junction, deep grey matter and upper brainstem, isolated DWI signal abnormalities of the corpus callosum may also be found. The splenium and the undersurface of the posterior body are mainly involved (Fig. 10) [31] due to the presence of the falx cerebri.

Signal abnormalities are thought to be due to traumatic axonal stretching with consequent depolarisation and cellular swelling [31]. ADC abnormalities may also represent Wallerian degeneration secondary to proximal neuronal death or axonal disconnection [29] and may therefore appear in the subacute phase. fronto-parietal white matter (arrows). ADC maps (b) presented reduced diffusivity. The neurological deficits and the DWI/ADC abnormalities resolved completely within 1 week, while slight T2 hyperintensities persisted on follow-up examinations
Most DAI lesions ( $80 \%)$ are microscopic [31] so that those visible on CT or MRI are just the tip of the iceberg: this fact might explain the poor correlation between neuroimaging and the clinical picture after a head trauma [32].

Demyelinating disorders

Multiple sclerosis (MS) is a chronic, multifocal, immunomediated inflammatory demyelinating and degenerative disorder of the central nervous system of unknown aetiology. MS lesions, known as plaques, typically appear as periventricular, ovoid white matter abnormalities perpendicular to the ventricles, along the path of the deep medullary veins.

On MRI the plaques are T2 hyperintense and show variable contrast enhancement. DWI usually discloses high $\mathrm{ADC}$ values in the plaque core due to prevailing inflammatory changes with vasogenic oedema or gliosis with myelin and axonal loss. Reduced ADC values may be found peripherally in acute MS lesions (Fig. 11) [33]. Electron microscopy studies have shown the presence of
Fig. 10 Axial MR images obtained in a child with a head injury at the level of the lateral ventricles. The midline of the splenium of the corpus callosum presented diffusion-weighted (a) and T2-weighted (c) hyperintensity (arrows) with reduced $\mathrm{ADC}$ values on $\mathrm{ADC}$ maps (b) consistent with diffuse axonal injury

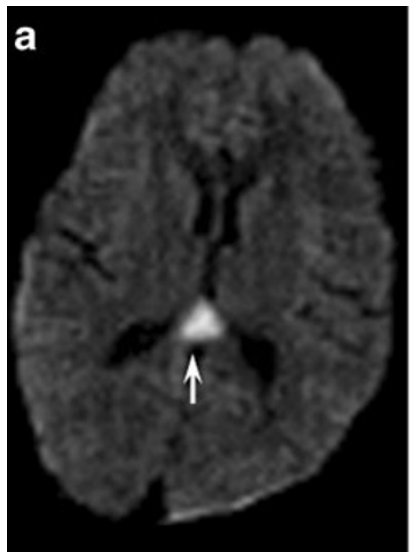

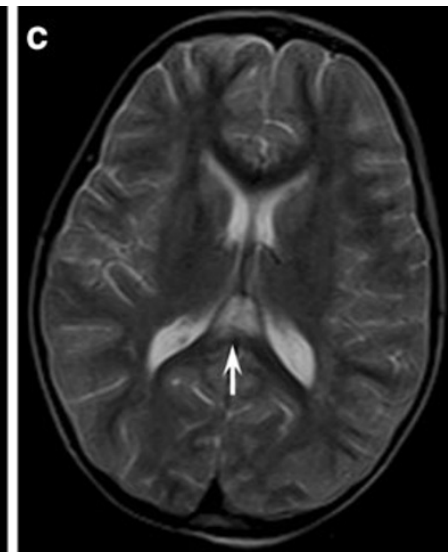


Fig. 11 A 30-year-old female patient affected by relapsingremitting multiple sclerosis (MS) presenting with a large demyelinating plaque in the right centrum semiovale. This atypical MS lesion appears hyperintense on diffusionweighted (a) and T2-weighted (c) axial images with predominant $\mathrm{ADC}$ restriction (b)
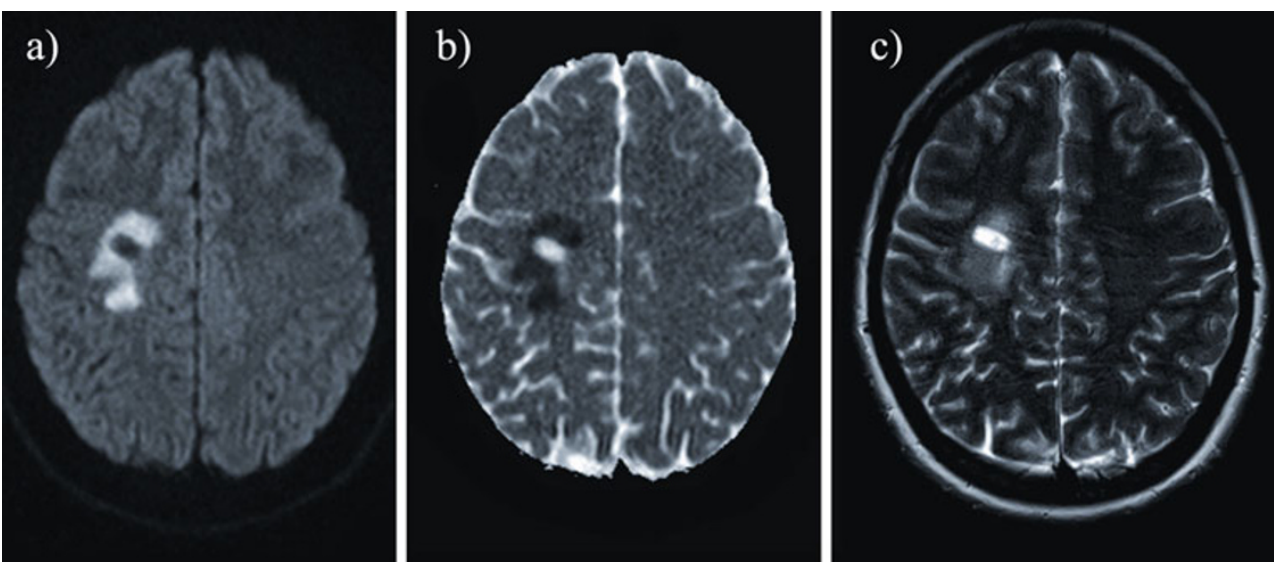

inflammatory infiltrates of lymphocytes and lipid-engulfing macrophages, swollen oligodendroglia, which could account for the ADC decrease [33]. Very rarely, acute plaques might present a homogeneous reduction in ADC values, which could suggest a diagnosis of stroke rather than an acute episode of MS [34]. However, according to our experience, concomitant lesions with increased ADC values are typically detected in these cases [34] and the cytotoxiclike lesions usually present components of increased ADC values as well $[33,34]$.

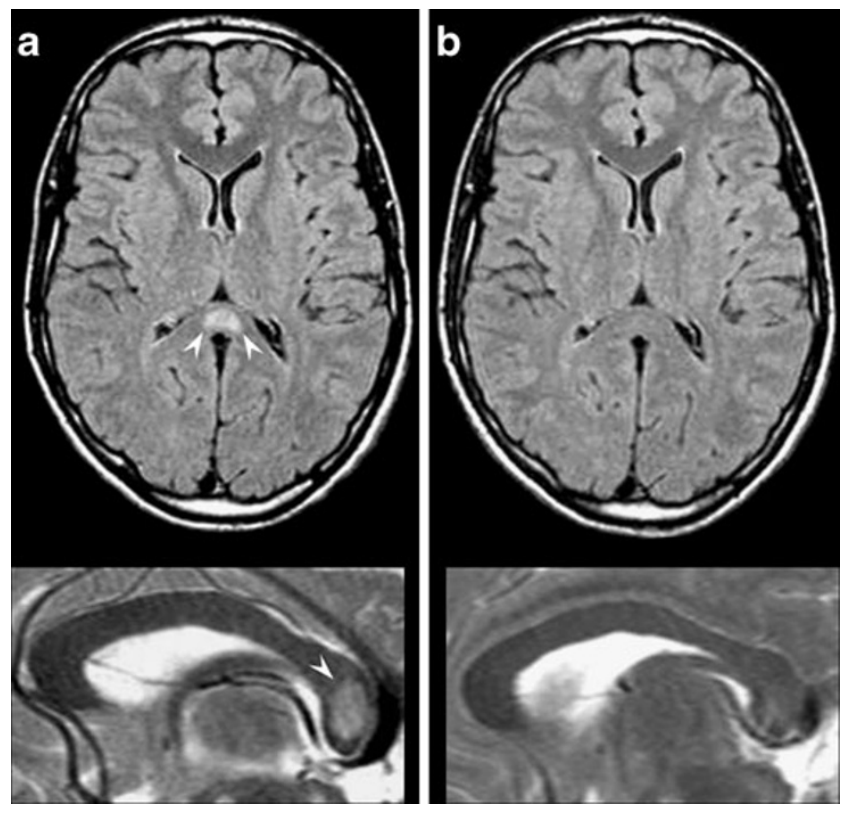

Fig. 12 A 32-year-old male patient admitted with partial seizures secondary to antiepileptic drug withdrawal. Axial FLAIR (superior row) and sagittal T2-weighted images (inferior row). At onset MRI examination (a) revealed increased signal intensity in the central area of the splenium of the corpus callosum (white arrowheads); 1 month later (b) the lesion had disappeared
Other conditions

Focal reversible lesions of the splenium (RSL) of the corpus callosum (Fig. 12) have been recently reported in several miscellaneous conditions such as viral encephalitis, encephalopathies, seizures, antiepileptic and anticancer drug administration [35, 36]. A few DWI studies [36, 37] have shown transitory restricted diffusion, but its aetiology and pathophysiology remain enigmatic. As a matter of fact, the proposed excitotoxic mechanism due to increased extracellular glutamate with consequent intramyelinic oedema [36] does not fully explain its detection in poorly myelinated brain such as in newborns with mitochondrial genetic disorders [38].

Clinically, RSL can present with seizures, confusion, ataxia, drowsiness, coma, headache and delirium, while the expected, specifically splenium-related deficits (visual simultanagnosia, hemialexia, unilateral agraphia and unilateral apraxia) are often difficult to evaluate.

Isolated corpus callosum lesions without concomitant parenchymal lesions usually regress rapidly, leading to complete clinical and neuroradiological recovery regardless of their cause $[35,36]$.

\section{Conclusions}

The literature ripens with diversified causes of areas of $\mathrm{ADC}$ restriction in the brain parenchyma: a few genetic, metabolic, iatrogenic, traumatic, infectious and vascular conditions can present with selective involvement of white matter with DWI hyperintense lesions and decreased ADC values. The neuroradiologist is often unnerved by the diagnostic challenges suggested by the clinician, as clinical history and course are not always disease-specific. In this setting, some suggestive or specific DWI MR patterns may provide powerful clues that can help in addressing the diagnosis and in tailoring treatment (Table 1). 


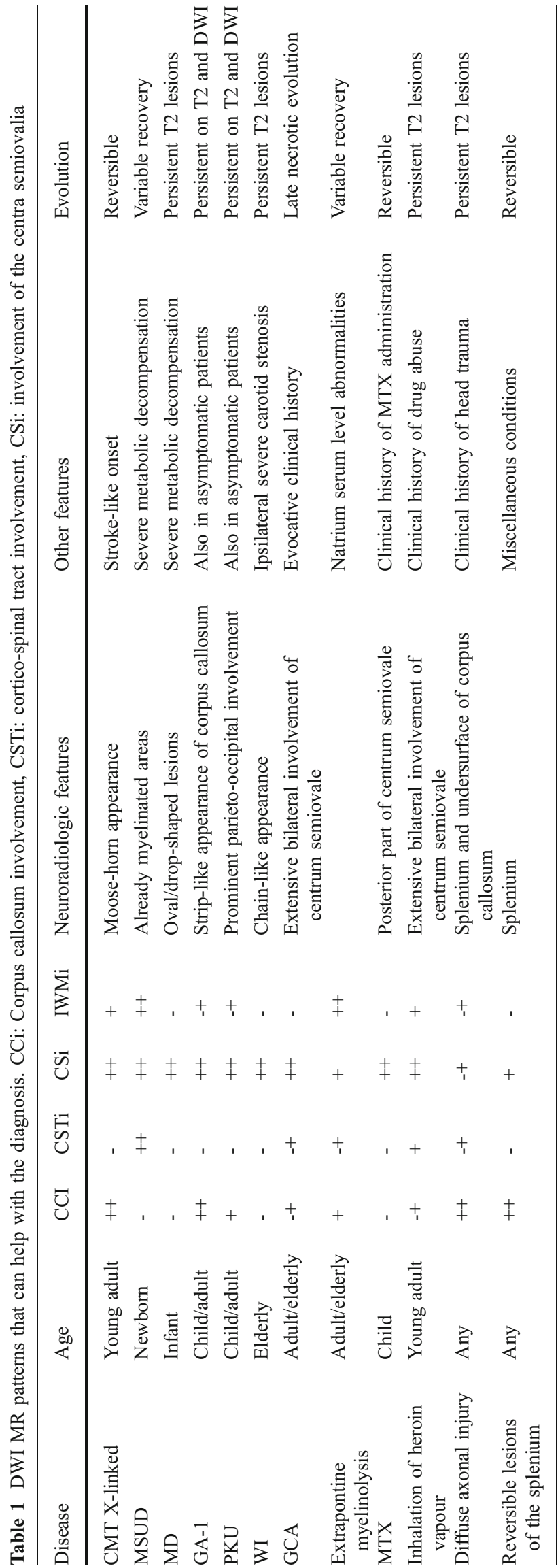

\section{References}

1. Schelhaas HJ, Van Engelen BG, Gabreëls-Festen AA, Hageman G, Vliegen JH, Van Der Knaap MS, Zwarts MJ (2002) Transient cerebral white matter lesions in a patient with connexin 32 missense mutation. Neurology 59:2007-2008

2. Hanemann CO, Bergmann C, Senderek J, Zerres K, Sperfeld AD (2003) Transient, recurrent, white matter lesions in X-linked Charcot-Marie-Tooth disease with novel connexin 32 mutation. Arch Neurol 60:605-609

3. Taylor RA, Simon EM, Marks HG, Scherer SS (2003) The CNS phenotype of X-linked Charcot-Marie-Tooth disease: more than a peripheral problem. Neurology 61:1475-1478

4. Van der Knaap MS, Valk J (2005) Maple Syrup Urine Disease. Magnetic Resonance of Myelination and Myelin Disorders. Springer, Heidelberg, Berlin New York, pp 311-319

5. Santos LM, Teixeira CD, Vilanova LC et al (2011) Menkes disease: case report of an uncommon presentation with white matter lesions. Arq Neuropsiquiatr 59:125-127

6. Blaser SI, Berns DH, Ross JS, Lanska MJ, Weissman BM (1989) Serial MR studies in Menkes disease. J Comput Assist Tomogr 13:113-115

7. Lee ES, Ryoo JW, Choi DS, Cho JM, Kwon SH, Shin HS (2007) Diffusion-weighted MR imaging of unusual white matter lesion in a patient with Menkes disease. Korean J Radiol 8:82-85

8. Hsich GE, Robertson RL, Irons M, Soul JS, du Plessis AJ (2000) Cerebral infarction in Menkes' disease. Pediatr Neurol 23:425-428

9. Kaler SG (1994) Menkes disease. Adv Pediatr 41:263-304

10. Harting I, Neumaier-Probst E, Seitz A et al (2009) Dynamic changes of striatal and extrastriatal abnormalities in glutaric aciduria type I. Brain 132:1764-1782

11. Brismar J, Ozand PT (1994) CT and MR of the brain in the diagnosis of organic acidemias. Experiences from 107 patients. Brain Dev 16:104-124

12. Dezortova M, Hajek M, Tintera J, Hejcmanova L, Sykova E (2001) MR in phenylketonuria-related brain lesions. Acta Radiol 42:459-466

13. Koch R, Moats R, Guttler F, Guldberg P, Nelson MJ (2000) Bloodbrain phenylalanine relationships in persons with phenylketonuria. Pediatrics 106:1093-1096

14. Vermathen P, Robert-Tissot L, Pietz J, Lutz T, Boesch C, Kreis R (2007) Characterization of white matter alterations in phenylketonuria by magnetic resonance relaxometry and diffusion tensor imaging. Magn Reson Med 58:1145-1156

15. Phillips MD, McGraw P, Lowe MJ, Mathews VP, Hainline BE (2001) Diffusion-weighted imaging of white matter abnormalities in patients with phenylketonuria. AJNR Am J Neuroradiol 22:1583-1586

16. Citton V, Manara R, Burlina A et al (2007) Diffusion weighted imaging (DWI) and white matter abnormalities (WMa) in earlytreated patients with phenylketonuria (PK). Neuroradiol 49:S75S162

17. Osborn AG (1994) Stroke. In: Osborn AG (ed) Diagnostic Neuroradiology. Mosby, St. Louis

18. Marcoux FW, Morawetz RB, Crowell RM, DeGirolami U, Halsey JH Jr (1982) Differential regional vulnerability in transient focal cerebral ischemia. Stroke 13:339-346

19. Hirano A, Zimmerman HM (1971) Some new pathological findings in the central myelinated axon. J Neuropathol Exp Neurol 30:325-336

20. Schwedemberg TH (1959) Leukoencephalopathy following carbon monoxide asphyxia. J Neuropathol Exp Neurol 18:597-608

21. Chalela JA, Wolf RL, Maldjian JA et al (2001) MRI identification of early white matter injury in anoxic-ischaemic encephalopathy. Neurology 56:481-485 
22. Ryan A, Molloy FM, Farrell MA et al (2005) Fatal toxic leukoencephalopathy: clinical, radiological and necropsy findings in two patients. J Neurol Neurosurg Psychiatry 76:10141016

23. McKinney AM, Teksam M, Felice R et al (2004) Diffusionweighted imaging in the setting of diffuse cortical laminar necrosis and hypoxic-ischemic encephalopathy. AJNR Am J Neuroradiol 25:1659-1665

24. Musson R, Romanowski C (2010) Restricted diffusion in Wallerian degeneration of the middle cerebellar peduncles following pontine infarction. Pol Przegl Radiol 75:38-43

25. Bermejo Garcés R, Cabada Giadas T, Bacaicoa Saralegui MC et al (2010) Bilateral wallerian degeneration of the pontocerebellar fibers secondary to pontine stroke: a report of 4 cases. Radiologia 52:71-75

26. Uchino A, Sawada A, Takase Y et al (2004) Symmetrical lesions of the middle cerebellar peduncle: MR imaging and differential diagnosis. Magn Reson Med Sci 3:133-140

27. Winick NJ, Bowman WP, Kamen BA et al (1992) Unexpected acute neurologic toxicity in the treatment of children with acute lymphoblastic leukemia. J Natl Cancer Inst 19:252-256

28. Mahoney DH Jr, Shuster JJ, Nitschke R et al (1998) Acute neurotoxicity in children with B-precursor acute lymphoid leukemia: an association with intermediate-dose intravenous methotrexate and intrathecal triple therapy - a Pediatric Oncology Group study. J Clin Oncol 16:1712-1722

29. Rubnitz JE, Relling MV, Harrison PL et al (1998) Transient encephalopathy following high-dose methotrexate treatment in childhood acute lymphoblastic leukemia. Leukemia 12:11761181

30. Keogh CF, Andrews GT, Spacey SD et al (2003) Neuroimaging features of heroin inhalation toxicity: "chasing the dragon.". AJR Am J Roentgenol 180:847-850

31. Osborn AG (1994) Diffuse axonal injury. In: Osborn AG (ed) Diagnostic Neuroradiology. Mosby, St. Louis

32. Scheid R, Walther K, Guthke T, Preul C, von Cramon DY (2006) Cognitive sequelae of diffuse axonal injury. Arch Neurol 63:418-424

33. Tievsky AL, Ptak T, Farkas J (1999) Investigation of apparent diffusion coefficient and diffusion tensor anisotrophy in acute and chronic multiple sclerosis lesions. AJNR Am J Neuroradiol 20:1491-1499

34. Rosso C, Remy P, Creange A, Brugieres P, Cesaro P, Hosseini H (2006) Diffusion-weighted MR imaging characteristics of an acute strokelike form of multiple sclerosis. AJNR Am J Neuroradiol 27:1006-1008

35. Gallucci M, Limbucci N, Paonessa A et al (2007) Reversible focal splenial lesions. Neuroradiology 49:541-544

36. Maeda M, Tsukahara $H$, Terada $H$ et al (2006) Reversible splenial lesion with restricted diffusion in a wide spectrum of diseases and conditions. J Neuroradiol 33:229-236

37. Bulakbasi N, Kocaoglu M, Tayfun C et al (2006) Transient splenial lesion of the corpus callosum in clinically mild influenzaassociated encephalitis/encephalopathy. AJNR Am J Neuroradiol 27:1983-1986

38. Takanashi J, Meda M, Hayashi M et al (2005) A neonate showing a reversible splenial lesion. Arch Neurol 62:1481-1882 